\title{
The Boomerang Attacks on the Round-Reduced Skein-512 *
}

\author{
Hongbo $\mathrm{Yu}^{1}$, Jiazhe $\mathrm{Chen}^{3}$, and Xiaoyun $\mathrm{Wang}^{2,3}$ \\ 1 Department of Computer Science and Technology, Tsinghua University, Beijing 100084, China \\ ${ }^{2}$ Institute for Advanced Study, Tsinghua University, Beijing 100084, China \\ \{yuhongbo, xiaoyunwang\}@mail.tsinghua.edu.cn \\ 3 Key Laboratory of Cryptologic Technology and Information Security, \\ Ministry of Education, School of Mathematics, Shandong University, Jinan 250100, China \\ jiazhechen@mail.sdu.edu.cn
}

\begin{abstract}
The hash function Skein is one of the five finalists of the NIST SHA-3 competition; it is based on the block cipher Threefish which only uses three primitive operations: modular addition, rotation and bitwise XOR (ARX). This paper studies the boomerang attacks on Skein-512. Boomerang distinguishers on the compression function reduced to 32 and 36 rounds are proposed, with complexities $2^{104.5}$ and $2^{454}$ respectively. Examples of the distinguishers on 28-round and 31round are also given. In addition, the boomerang distinguishers are applicable to the key-recovery attacks on reduced Threefish-512. The complexities for key-recovery attacks reduced to 32-/33/34-round are about $2^{181}, 2^{305}$ and $2^{424}$. Because Laurent et al. [14] pointed out that the previous boomerang distinguishers for Threefish-512 are in fact not compatible, our attacks are the first valid boomerang attacks for the final round Skein-512.
\end{abstract}

Key words: Hash function, Boomerang attack, Threefish, Skein

\section{Introduction}

Cryptographic hash functions, which provide integrity, authentication and etc., are very important in modern cryptology. In 2005, as the most widely used hash functions MD5 and SHA-1 were broken by Wang et al. [18][19], NIST started a hash competition for a new hash standard (SHA-3) in 2007. Now the competition has come into the third round (the final round), and 5 out of the candidates are selected. Skein [7], which is one of the finalists, is a ARX-type hash function (based on modular addition, rotation and exclusive-OR). The core of the compression function of Skein is a tweakable block cipher called Threefish, which is proposed with 256-, 512-, 1024-bit block sizes and 72, 72, 80 rounds, respectively. When the algorithm entered into the second round, the authors had changed the rotation constants, and after it was selected as a finalist, the constants used in the key schedule were updated to resist the rotational attack $[10,11]$.

During the competition, Skein has been attracting the attentions of the cryptanalysts, and there are several cryptanalytic results on the security of the compression function of Skein and its based block cipher Threefish. At Asiacrypt 2009 [1], Aumasson et al. used the boomerang attack to launch a key recovery attack on Threefish-512 reduced to 32 rounds and the knownkey distinguisher to 35 rounds under the old rotation constants. However, we find that their differential paths use an inverse permutation instead of the original one. At ISPEC 2010 [6], Chen et al. also proposed a boomerang attack for the key recovery of Threefish-512 reduced to 33 and 34 rounds. At CT-RSA 2012 [14], Leurent et al. gave a boomerang distinguisher for 32-round compression function of Skein-256 with complexity $2^{114}$, and they also pointed that the differential paths in [6] are incompatible. We correct the paths in [1] with the right permutation and show that they are also incompatible under the old rotation constants due to similar contradictions as in [6]. At CANS 2010 [15], Su et al. presented free-start near-collisions of Skein-256/-512 compression functions reduced to 20 rounds and Skein-1024 reduced to 24 rounds.

* Supported by the National Natural Science Foundation of China (No.61133013) and the Tsinghua University Initiative Scientific Research Program (No.20111080970). 
At Asiacrypt 2010 [11], Khovratovich et al. combined the rotational attack and the rebound attack, and gave distinguishers on 53-round Skein-256 and 57-round Skein-512 respectively, and their technique depends on the constants used in the key schedule. In 2011, Yu et al. [20] gave a near-collision attack for Skein-256 using the rebound attack. At FSE 2012, Khovratovich et al. also gave a preimage attack on 22-round Skein-512 hash function and 37-round Skein-512 compression function by the biclique method [12].

Our contribution. In this paper, we study the boomerang distinguishers on round-reduced Skein-512. Our analysis is based on two related-key differential paths of Threefish-512 with high probability. In order to get rid of the incompatibility pointed out in [14], we use differences for the key words and tweaks on bit 59 instead of the bit 64 (the bit 1 is the least significant bit) for the top path.

We also reveal that the four paths in the middle 8 rounds are not independent, the probability of the distinguisher in the middle 8 rounds is much higher than the average probability. Based on the differential paths, we give boomerang distinguisher on the compression function of Skein512 reduced to 32 round with complexity $2^{104.5}$. The distinguisher can be extended to 36 rounds by adding two more rounds on the top and bottom of the differential paths respectively. Our boomerang distinguishers also can be used to the related-key key-recovery attack on Threefish512 reduced to 32,33 and 34 rounds for $1 / 4$ of the keys. Table 1 summaries our results.

The rest of the paper is organized as follows. In Sect.2, we give a brief description of Skein512. Sect.3 summaries the boomerang attack. Sect.4 leverages the boomerang technique to the compression functions of Skein-512. In Sec.5, we introduce the key-recovery attacks based on our boomerang distinguishers. Finally, a conclusion of the paper is given in Sect.6.

Table 1. Summary of the attacks on Skein (only the attacks independent of the constants are mentioned).

\begin{tabular}{|l|c|c|c|c|}
\hline Attack & CF $/$ KP & Rounds & Time & Ref. \\
\hline \hline Near collisions(Skein-256) & CF & 20 & $2^{60}$ & {$[15]$} \\
\hline Near Collisions(Skein-256) & CF & 32 & $2^{105}$ & {$[20]$} \\
\hline Boomerang Dist.(Skein-256) & CF & 28 & $2^{24}$ & \\
Boomerang Dist.(Skein-256) & KP & 32 & $2^{57}$ & {$[14]$} \\
Boomerang Dist.(Skein-256) & CF & 32 & $2^{114}$ & \\
\hline \hline Key Recovery (Threefish-512) & KP & 32 & $2^{312}$ & \multirow{2}{*}[1]{$^{*}$} \\
Boomerang Dist. (Threefish-512) & KP & 35 & $2^{478}$ & \\
\hline Key Recovery (Threefish-512) & KP & 32 & $2^{189}$ & \\
Key Recovery (Threefish-512) & KP & 33 & $2^{324.6}$ & \multirow{2}{*}[6]{$^{*}$} \\
Key Recovery (Threefish-512) & KP & 34 & $2^{474.4}$ & \\
\hline \hline Boomerang Dist.(Skein-512) & CF & 28 & $2^{40.5}$ & \\
Boomerang Dist.(Skein-512) & CF & 31 & $2^{32} \dagger$ & \\
Boomerang Dist.(Skein-512) & CF & 32 & $2^{56.5} \dagger$ & \\
Boomerang Dist.(Skein-512) & CF & 32 & $2^{104.5}$ & \multirow{2}{*}{ Sec.3 } \\
Boomerang Dist.(Skein-512) & CF & 33 & $2^{125} \dagger$ & \\
Boomerang Dist.(Skein-512) & CP & 34 & $2^{190.6} \dagger$ & \\
Boomerang Dist.(Skein-512) & CP & 35 & $2^{308} \dagger$ & \\
Boomerang Dist.(Skein-512) & CP & 36 & $2^{454} \dagger$ & \\
\hline Key-recovery (Threefish-512) & KP & 32 & $2^{181}$ & \\
Key-recovery (Threefish-512) & KP & 33 & $2^{305}$ & Sec.4 \\
Key-recovery (Threefish-512) & KP & 34 & $2^{424}$ & \\
\hline KP: Keyed permutation, CF: Compression Function & \\
*:The differential paths are incompatible & & \\
$\dagger$ \\
†: The initial and final subkeys are not included & & \\
\hline
\end{tabular}




\section{Description of Skein-512}

Skein is designed by Ferguson et al., which is one of the SHA-3 finalists. It supports three different internal state sizes $(256,512$, and 1024 bits) and each of these state sizes can support any output size. The word size which Skein operates on is 64 bits. Skein is based on the UBI (Unique Block Iteration) chaining mode that uses block cipher Threefish to build a compression function.

The compression function of Skein can be defined as $H=E(K, T, M) \oplus M$, where $E(K, T, M)$ is the block cipher Threefish, $M$ is the plaintext, $K$ is the master key and $T$ is the tweak value. For Skein-512, both $M$ and $K$ are 512 bits, and the length of $T$ is 128 bits. Let us denote $V_{i}=\left(a_{i}, b_{i}, c_{i}, d_{i}, e_{i}, f_{i}, g_{i}, h_{i}\right)$ as the output value of the $i$-th round, where $a_{i}, b_{i}, \ldots, h_{i}$ are 64 -bit words. Let $V_{0}=M$ be the plaintext, the encryption procedure of Threefish-512 is carried out for $i=1$ to 72 as follows.

If $(i-1) \bmod 4=0$, first compute

$$
\begin{aligned}
& \hat{a}_{i-1}=a_{i-1}+K_{(i-1) / 4, a}, \hat{b}_{i-1}=b_{i-1}+K_{(i-1) / 4, b}, \\
& \hat{c}_{i-1}=c_{i-1}+K_{(i-1) / 4, c}, \hat{d}_{i-1}=d_{i-1}+K_{(i-1) / 4, d}, \\
& \hat{e}_{i-1}=e_{i-1}+K_{(i-1) / 4, e}, \hat{f}_{i-1}=f_{i-1}+K_{(i-1) / 4, f}, \\
& \hat{g}_{i-1}=g_{i-1}+K_{(i-1) / 4, g}, \hat{h}_{i-1}=h_{i-1}+K_{(i-1) / 4, h},
\end{aligned}
$$

where $K_{(i-1) / 4, a}, K_{(i-1) / 4, b}, \ldots, K_{(i-1) / 4, h}$ are round subkeys which are involved in every four rounds. Then carry out:

$$
\begin{aligned}
a_{i} & =\hat{c}_{i-1}+\hat{d}_{i-1}, h_{i}=a_{i} \oplus\left(\hat{d}_{i-1} \lll R_{i, 1}\right), \\
c_{i} & =\hat{e}_{i-1}+\hat{f}_{i-1}, f_{i}=c_{i} \oplus\left(\hat{f}_{i-1} \lll R_{i, 2}\right), \\
e_{i} & =\hat{g}_{i-1}+\hat{h}_{i-1}, d_{i}=e_{i} \oplus\left(\hat{h}_{i-1} \lll R_{i, 3}\right), \\
g_{i} & =\hat{a}_{i-1}+\hat{b}_{i-1}, b_{i}=g_{i} \oplus\left(\hat{b}_{i-1} \lll R_{i, 0}\right),
\end{aligned}
$$

where $R_{i, 1}$ and $R_{i, 2}$ are rotation constants which can be found in [7]. For the sake of convenience, we denote $\hat{V}_{i-1}=\left(\hat{a}_{i-1}, \hat{b}_{i-1}, \hat{c}_{i-1}, \hat{d}_{i-1}, \hat{e}_{i-1}, \hat{f}_{i-1}, \hat{g}_{i-1}, \hat{h}_{i-1}\right)$.

If $(i-1) \bmod 4 \neq 0$, compute

$$
\begin{gathered}
a_{i}=c_{i-1}+d_{i-1}, h_{i}=a_{i} \oplus\left(d_{i-1} \lll R_{i, 1}\right), \\
c_{i}=e_{i-1}+f_{i-1}, f_{i}=c_{i} \oplus\left(f_{i-1} \lll R_{i, 2}\right), \\
e_{i}=g_{i-1}+h_{i-1}, d_{i}=e_{i} \oplus\left(h_{i-1} \lll R_{i, 3}\right), \\
g_{i}=a_{i-1}+b_{i-1}, b_{i}=g_{i} \oplus\left(b_{i-1} \lll R_{i, 0}\right) .
\end{gathered}
$$

After the last round, the ciphertext is computed as $\hat{V}_{72}=\left(\hat{a}_{72}, \hat{b}_{72}, \hat{c}_{72}, \hat{d}_{72}, \hat{e}_{72}, \hat{f}_{72}, \hat{g}_{72}, \hat{h}_{72}\right)$.

The key schedule starts with the master key $K=\left(k_{0}, k_{1}, k_{2}, k_{3}, k_{4}, k_{5}, k_{6}, k_{7}\right)$ and the tweak value $T=\left(t_{0}, t_{1}\right)$. First we compute

$$
k_{8}:=0 x 1 b d 11 b d a a 9 f c 1 a 22 \oplus \bigoplus_{i=0}^{7} k_{i} \quad \text { and } \quad t_{2}:=t_{0} \oplus t_{1} .
$$

Then the subkeys are derived for $s=0$ to 18 :

$$
\begin{aligned}
& K_{s, a}:=k_{(s+0) \bmod 9} \\
& K_{s, b}:=k_{(s+1) \bmod 9} \\
& K_{s, c}:=k_{(s+2) \bmod 9} \\
& K_{s, d}:=k_{(s+3) \bmod 9} \\
& K_{s, e}:=k_{(s+4) \bmod 9} \\
& K_{s, f}:=k_{(s+5) \bmod 9}+t_{s \bmod 3} \\
& K_{s, g}:=k_{(s+6) \bmod 5}+t_{(s+1) \bmod 3} \\
& K_{s, h}:=k_{(s+7) \bmod 5}+s
\end{aligned}
$$




\section{The Boomerang Attack}

The boomerang attack was introduced by Wagner [16] and first applied to block ciphers; it is an adaptive chosen plaintext and ciphertext attack. Later it was further developed by Kelsey et al. into a chosen plaintext attack called the amplified boomerang attack [13], then Biham et al. further developed it into the rectangle attack [3]. The basic idea of the boomerang attack is joining two short differential paths with high probabilities in a quartet. The related-key boomerang attack is proposed in [4]; it uses the related-key differentials instead of the single-key differentials. Let $E$ be a block cipher with block size $n$ bits, and it can be decomposed into two sub-ciphers: $E=E_{1} \circ E_{0}$. For the sub-cipher $E_{0}$, there is a differential path $\left(\alpha, \alpha_{k}\right) \rightarrow \beta$ with probability $p$; for the sub-cipher $E_{1}$, there is a differential path $\left(\gamma, \gamma_{k}\right) \rightarrow \delta$ with probability $q$. Then the related-key boomerang attack can be constructed:

- Randomly choose a pair of plaintexts $\left(P_{1}, P_{2}\right)$ such that $P_{2}-P_{1}=\alpha$.

- Compute $\mathcal{K}_{2}=\mathcal{K}_{1}+\alpha_{k}, \mathcal{K}_{3}=\mathcal{K}_{1}+\gamma_{k}$ and $\mathcal{K}_{4}=\mathcal{K}_{1}+\alpha_{k}+\gamma_{k}$. Encrypt $P_{1}, P_{2}$ with the related keys $\mathcal{K}_{1}$ and $\mathcal{K}_{2}$ to get $C_{1}=E_{\mathcal{K}_{1}}\left(P_{1}\right), C_{2}=E_{\mathcal{K}_{2}}\left(P_{2}\right)$.

- Compute $C_{3}=C_{1}+\delta, C_{4}=C_{2}+\delta$. Decrypt $C_{3}, C_{4}$ with the related keys $\mathcal{K}_{3}$ and $\mathcal{K}_{4}$ to get $P_{3}=E_{\mathcal{K}_{3}}^{-1}\left(C_{3}\right), P_{4}=E_{\mathcal{K}_{4}}^{-1}\left(C_{4}\right)$

- Check whether $P_{4}-P_{3}=\alpha$.

It is known that for a $n$-bit random permutation, $P_{4}-P_{3}=\alpha$ with probability $2^{-n}$. Therefore, the attack is valid if $p^{2} q^{2}>2^{-n}$.

In the known-key setting, a (related-key) boomerang attack can be used to distinguish a given permutation from a random oracle; it is called known-related-key boomerang attack in [5]. Applying the known-related-key boomerang attack to the compression function in the MMO mode, i.e, $C F(K, M)=E_{K}(M)+M$, it is possible to start from the middle rounds because the message $M$ and the key $K$ can be selected randomly (refer to [5] and [14]). The (known-relatedkey) boomerang attack is particularly efficient for the ARX-type hash functions because their compression functions have strong diffusion after several steps, only short differential paths with high probabilities can be found. See Fig. 1 for the schematic view of the boomerang distinguisher for hash functions. The known-related-key boomerang attack for a permutation (or a compression function in the MMO structure) can be summarized as follows.

- Choose a random value $X_{1}$ and $\mathcal{K}_{1}$, compute $X_{2}=X_{1}+\beta, X_{3}=X_{1}+\gamma, X_{4}=X_{3}+\beta$ and $\mathcal{K}_{2}=\mathcal{K}_{1}+\beta_{k}, \mathcal{K}_{3}=\mathcal{K}_{1}+\gamma_{k}, \mathcal{K}_{4}=\mathcal{K}_{3}+\beta_{k}$.

- Compute backward from quartets $\left(X_{i}, \mathcal{K}_{i}\right)_{i=1}^{4}$ using $E_{0}^{-1}$ to obtain $P_{1}, P_{2}, P_{3}$ and $P_{4}$.

- Compute forward from quartets $\left(X_{i}, \mathcal{K}_{i}\right)_{i=1}^{4}$ using $E_{1}$ to obtain $C_{1}, C_{2}, C_{3}$ and $C_{4}$.

- Check whether $P_{2}-P_{1}=P_{4}-P_{3}=\alpha$ and $C_{3}-C_{1}=C_{4}-C_{2}=\delta$ are fulfilled.

For a $n$-bit random permutation, the boomerang distinguisher falls into three types according to the input and output differences.

- Type I: A quartet satisfies $P_{2}-P_{1}=P_{4}-P_{3}=\alpha$ and $C_{3}-C_{1}=C_{4}-C_{2}=\delta$ for fixed $\alpha$ and $\delta$. In this case, the generic complexity is $2^{n}$.

- Type II: Only $C_{3}-C_{1}=C_{4}-C_{2}$ are required (the property is also called zero-sum or second-order differential collision). In this case, the complexity for obtaining such a quartet is $2^{n / 3}$ using Wagner's generalized birthday attack [17].

- Type III: A quartet satisfies $P_{2}-P_{1}=P_{4}-P_{3}$ and $C_{3}-C_{1}=C_{4}-C_{2}$. In this case, the best known attack still takes time $2^{n / 2}$. 


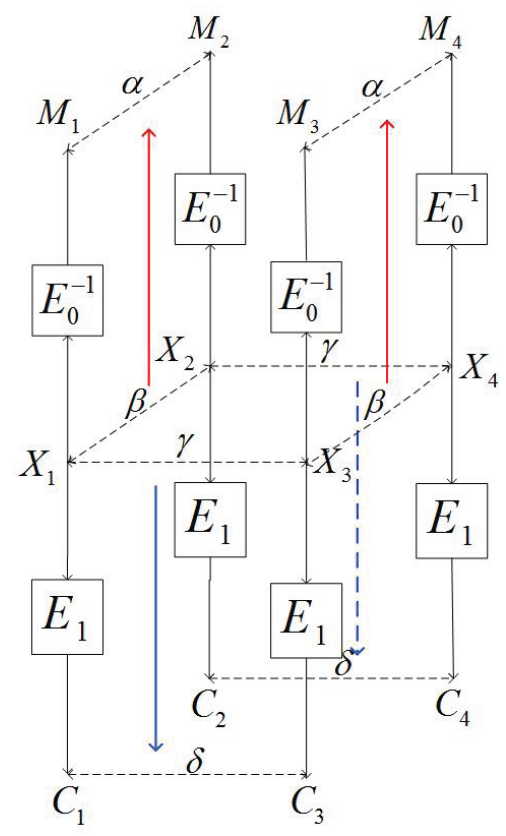

Fig. 1. The boomerang attack for the hash functions

\section{The Boomerang Distinguisher on Reduced Skein-512}

In this section, we describe the known-related-key boomerang attack on Skein-512 reduced to 36 rounds. As mentioned above, the basic idea of our attack is to connect two short differential paths in a quartet. The first step of our attack is to find two short differentials with high probabilities so that the switch in the middle does not contain any contradictions. Secondly, we derive the sufficient conditions for the rounds in the middle, and compute the precise probability of each condition. Thirdly, we correct the conditions in the intermediate rounds by modifying the chaining variables, the key $K$ and the tweak value $T$. Finally, after the message modification, we search the right quartet that pass the verification of the distinguisher.

\subsection{Round-reduced differential paths for Skein-512}

The differences of the master key $K=\left(k_{i}\right)_{i=0}^{7}$ and tweak value $T=\left(t_{0}, t_{1}\right)$ selected for the top differential path are $\Delta k_{0}=0 x 0400000000000000, \Delta t_{0}=0 x 0400000000000000$ and $\Delta t_{1}=$ $0 x 0400000000000000$. Suppose $k_{8,59}=t_{0,59} \oplus 1$ and $k_{0,59}=t_{1,59} \oplus 1$, then there is no difference in the fourth subkey. For the bottom path, the MSB differences are set in $k_{3}, k_{4}$ and $t_{1}$, and this gives no difference in the eighth subkey. According to the key schedule, the differences for the subkeys $K_{i}=\left(K_{i, a}, K_{i, b}, K_{i, c}, K_{i, d}, K_{i, e}, K_{i, f}, K_{i, g}, K_{i, h}\right)(0 \leq i \leq 9)$ are shown in Tables 2 and 3.

The top path we used consists of 18 rounds. Because $\Delta K_{2}=\left(0,0,0,0,0,0,0, \pm 2^{58}\right)$ and $\Delta K_{3}=(0,0,0,0,0,0,0,0)$, we select the intermediate difference $\Delta V_{8}$ to meet $\left(0,0,0,0,0,0,0, \mp 2^{58}\right)$. In this way, we get a 8-round path with zero-difference from rounds 9 to 16 . By extending the difference $\Delta V_{8}$ in the backward direction for 6 rounds and the difference $\Delta \hat{V}_{16}=\Delta K_{4}$ in the forward direction for 4 rounds, a 18-round differential path with high probability is obtained.

Similarly, we choose $\Delta V_{24}$ as $\left(0,0,0,0,0,0,0,2^{63}\right)$ to compensate the difference $\Delta K_{6}=$ $\left(0,0,0,0,0,0,0,2^{63}\right)$, which results in zero difference in rounds 25 to 32 . As a consequence, a 18-round differential path with high probability also can be acquired by linearly expanding the difference $\Delta V_{24}$ backward for 4 rounds and the difference $\Delta \hat{V}_{32}=\Delta K_{8}$ forward for 6 rounds. 
Table 2. The subkey differences of the top path.

\begin{tabular}{|c|c|c|c|c|c|c|c|c|}
\hline $\mathrm{s} d$ & $K_{i, a}$ & $K_{i, b}$ & $K_{i, c}$ & & $\begin{array}{r}K_{i, e} \\
\text { fferen }\end{array}$ & $\begin{array}{l}K_{i, f} \\
\text { ces }\end{array}$ & $K_{i, g}$ & $K_{i, h}$ \\
\hline $\begin{array}{lll}0 & 0\end{array}$ & $\begin{array}{c}k_{0} \\
\pm 2^{58}\end{array}$ & $\begin{array}{c}k_{1} \\
0\end{array}$ & $\begin{array}{c}k_{2} \\
0\end{array}$ & $\begin{array}{c}k_{3} \\
0\end{array}$ & $\begin{array}{c}k_{4} \\
0\end{array}$ & $\begin{array}{c}k_{5}+t_{0} \\
\pm 2^{58}\end{array}$ & $\begin{array}{c}k_{6}+t_{1} \\
\pm 2^{58}\end{array}$ & $\begin{array}{c}k_{7}+0 \\
0\end{array}$ \\
\hline 14 & $\begin{array}{c}k_{1} \\
0 \\
\end{array}$ & $\begin{array}{c}k_{2} \\
0 \\
\end{array}$ & $\begin{array}{c}k_{3} \\
0 \\
\end{array}$ & $\begin{array}{c}k_{4} \\
0 \\
\end{array}$ & $\begin{array}{c}k_{5} \\
0 \\
\end{array}$ & $\begin{array}{c}k_{6}+t_{1} \\
\pm 2^{58}\end{array}$ & $\begin{array}{c}k_{7}+t_{2} \\
0 \\
\end{array}$ & $\begin{array}{c}k_{8}+1 \\
\pm 2^{58}\end{array}$ \\
\hline 28 & $\begin{array}{c}k_{2} \\
0\end{array}$ & $\begin{array}{c}k_{3} \\
0\end{array}$ & $\begin{array}{c}k_{4} \\
0\end{array}$ & $\begin{array}{c}k_{5} \\
0\end{array}$ & $\begin{array}{c}k_{6} \\
0\end{array}$ & $\begin{array}{c}k_{7}+t_{2} \\
0\end{array}$ & $\begin{array}{c}k_{8}+t_{0} \\
0\end{array}$ & $\begin{array}{c}k_{0}+2 \\
\pm 2^{58}\end{array}$ \\
\hline $\begin{array}{ll}312 \\
\end{array}$ & $\begin{array}{c}k_{3} \\
0\end{array}$ & $\begin{array}{c}k_{4} \\
0\end{array}$ & $\begin{array}{c}k_{5} \\
0\end{array}$ & $\begin{array}{c}k_{6} \\
0\end{array}$ & $\begin{array}{c}k_{7} \\
0\end{array}$ & $\begin{array}{c}k_{8}+t_{0} \\
0\end{array}$ & $\begin{array}{c}k_{0}+t_{1} \\
0\end{array}$ & $\begin{array}{c}k_{1}+3 \\
0\end{array}$ \\
\hline 416 & $\begin{array}{c}k_{4} \\
0 \\
\end{array}$ & $\begin{array}{c}k_{5} \\
0 \\
\end{array}$ & $\begin{array}{c}k_{6} \\
0 \\
\end{array}$ & $\begin{array}{c}k_{7} \\
0\end{array}$ & $\begin{array}{c}k_{8} \\
\pm 2^{58}\end{array}$ & $\begin{array}{c}k_{0}+t_{1} \\
0\end{array}$ & $\begin{array}{c}k_{1}+t_{2} \\
0\end{array}$ & $\begin{array}{c}k_{2}+4 \\
0\end{array}$ \\
\hline
\end{tabular}

Table 3. The subkey differences of the bottom path.

\begin{tabular}{|c|c|c|c|c|c|c|c|c|}
\hline s d & $K_{i, a}$ & $K_{i, b}$ & $K_{i, c}$ & & Differences & $K_{i, f}$ & $K_{i, g}$ & $K_{i, h}$ \\
\hline \multirow{2}{*}{520} & $k_{5}$ & $k_{6}$ & $k_{7}$ & $k_{8}$ & $k_{0}$ & $k_{1}+t_{2}$ & $k_{2}+t_{0}$ & $k_{3}+5$ \\
\hline & 0 & 0 & 0 & 0 & 0 & $2^{63}$ & 0 & $2^{63}$ \\
\hline \multirow{2}{*}{624} & $k_{6}$ & $k_{7}$ & $k_{8}$ & $k_{0}$ & $k_{1}$ & $k_{2}+t_{0}$ & $k_{3}+t_{1}$ & $k_{4}+6$ \\
\hline & 0 & 0 & 0 & 0 & 0 & 0 & 0 & $2^{63}$ \\
\hline \multirow{2}{*}{728} & $k_{7}$ & $k_{8}$ & $k_{0}$ & $k_{1}$ & $k_{2}$ & $k_{3}+t_{1}$ & $k_{4}+t_{2}$ & $k_{5}+7$ \\
\hline & 0 & 0 & 0 & 0 & 0 & 0 & 0 & 0 \\
\hline \multirow{2}{*}{832} & $k_{8}$ & $k_{0}$ & $k_{1}$ & $k_{2}$ & $k_{3}$ & $k_{4}+t_{2}$ & $k_{5}+t_{0}$ & $k_{6}+8$ \\
\hline & 0 & 0 & 0 & 0 & $2^{63}$ & 0 & 0 & 0 \\
\hline \multirow{2}{*}{936} & $k_{0}$ & $k_{1}$ & $k_{2}$ & $k_{3}$ & $k_{4}$ & $k_{5}+t_{0}$ & $k_{6}+t_{1}$ & $k_{7}+9$ \\
\hline & 0 & 0 & 0 & $2^{63}$ & $2^{63}$ & 0 & $2^{63}$ & 0 \\
\hline
\end{tabular}

The two differential paths are shown in Tables 10 and 11, where we use two kinds of differences: the XOR difference and the integer modular substraction difference. In the rounds after adding the subkey, we express the differences in the positions $\hat{a}_{i}, \hat{c}_{i}, \hat{e}_{i}$ and $\hat{g}_{i}$ with the integer modular substraction difference (except the final adding key round), because the XOR operations are not included when computing the next chaining value $V_{i+1}$; in the other positions of the differential path, we use the XOR difference.

\subsection{Message modifications for the middle rounds}

The conditions of the middle 8 rounds can be satisfied by the message modifications. The two pair short differentials in the boomerang distinguisher from rounds 16 to 24 are shown in Fig. 2. Let $D_{1}, D_{2}$ denote the top two paths from rounds 20 down to 16 , and $D_{3}, D_{4}$ be the bottom two paths from rounds 20 to 24 . Then the sufficient conditions for the four paths are shown in Table 4.

If we select the chaining variables $V_{20}^{(1)}$ and the subkey $K_{5}^{(1)}$ randomly, then the conditions in $D_{1}$ can be fulfilled by modifying $V_{20}^{(1)}$; those in $D_{3}$ can be satisfied by modifying $K_{5}^{(1)}$. But for conditions in $D_{2}$ and $D_{4}$, we cannot correct them directly because the pairs $\left(V_{20}^{(3)}, K_{5}^{(3)}\right)$ and $\left(V_{20}^{(2)}, K_{5}^{(2)}\right)$ are related to the pair $\left(V_{20}^{(1)}, K_{5}^{(1)}\right)$.

Let the non-zero difference bits of $V_{i}^{(3)}$ related to differential path $D_{2}$ equal to the corresponding bits of $V_{i}^{(1)}$ where $i=17,18,19,20$. That is to say $a_{20,9}^{(3)}=a_{20,9}^{(1)}, a_{20,34}^{(3)}=a_{20,34}^{(1)}$, $b_{20,39}^{(3)}=b_{20,39}^{(1)}, \cdots$ and so on (see Table 5 ). As a result, if all the sufficient conditions for the path $D_{1}$ are satisfied, then all the conditions in $D_{2}$ must be satisfied. For the fixed input difference $\gamma$ of $D_{3}$, We can easily deduce that the conditions in Table 5 can be satisfied with probability 


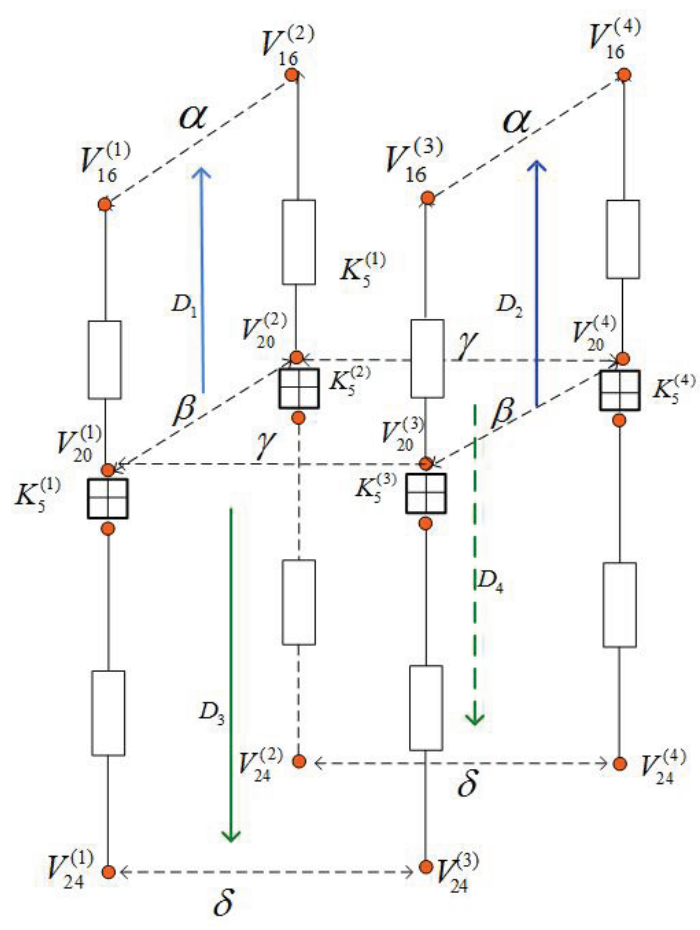

Fig. 2. The middle rounds in a boomerang distinguisher

$2^{-7.4}$, which is much higher than the average probability; this is also verified by our experiments. All the conditions in Table 5 can be fulfilled by modifying $V_{20}^{(1)}$.

Similarly, we can convert the conditions for $D_{4}$ in Table 4 to those in Table 6 . These conditions hold with probability $2^{-8.4}$ when $D_{1}$ hold, which is better than the average probability $2^{-32}$. All the conditions in Table 6 can be fulfilled by modifying $K_{5}^{(1)}$.

After the message modifications, the boomerang distinguisher in the middle 8 rounds hold with probability close to 1 . We also reveal that the differential path $D_{2}$ is heavily dependent on $D_{3}$, and the path $D_{4}$ is heavily dependent on $D_{1}$. The reason of contradictions in the previous attacks on Skein-512 is that the probability for $D_{2}$ or $D_{4}$ to hold is zero when the paths $D_{1}$ and $D_{3}$ hold.

\subsection{Complexity of the Attack}

Using the differential paths given in Table 10 and Table 11, we can construct a boomerang distinguisher for Skein-512 reduced to 32 rounds (out of 72 rounds). The top path in the backward direction (rounds 16-4) holds with probability $2^{-37}$ after the message modifications. The bottom path in the forward direction (rounds 20-36) holds with probability $2^{-24}$ after message modifications.

So the complexity of the 32-round boomerang distinguisher is $2^{2 \cdot(37+24)}=2^{122}$ by using the differential paths in Table 10 and 11. It can be reduced to $2^{2 \cdot(13+6)} \times 3^{24+18} \approx 2^{104.5}$ if we only want $\oplus_{i=1}^{4} P_{i}=0$ and $\oplus_{i=1}^{4} C_{i}=0$, because the probability for $\oplus_{i=1}^{4} x_{j}^{(i)}=0$ is about $1 / 3$ where $x_{j}^{(i)}$ denote the non-zero difference bits in rounds 4 and 36 . Extending the 32-round boomerang distinguisher for two more rounds, we can get the 33-/34-/35-/36-round boomerang distinguisher on Skein-512 as follows.

- The complexity of 33-round distinguisher (rounds $4-37$ ) is about $2^{2 \cdot(13+6)} \times 3^{24+13+18} \approx 2^{125}$. 
Table 4. The conditions for differential paths $D_{1}, D_{2}, D_{3}$ and $D_{4}$.

\begin{tabular}{|c|c|c|}
\hline unds & or $D_{1}$ & \\
\hline 20 & $\begin{array}{l}a_{20,43}^{(1)} \oplus h_{20,43}^{(1)}=a_{20,34}^{(1)}, c_{20,63}^{(1)} \oplus f_{20,63}^{(1)}=c_{20,9}^{(1)}, \\
c_{20,21}^{(1)} \oplus f_{20,21}^{(1)}=c_{20,31}^{(1)}, c_{20,35}^{(1)} \oplus f_{20,35}^{(1)}=c_{20,45}^{(1)}\end{array}$ &, $\begin{array}{l}a_{20,43}^{(3)} \oplus h_{20,43}^{(3)}=a_{20,34}^{(3)}, c_{20,63}^{(3)} \oplus f_{20,63}^{(3)}=c_{20,9}^{(3)}, \\
c_{20,21}^{(3)} \oplus f_{20,21}^{(3)}=c_{20,31}^{(3)}, c_{20,35}^{(3)} \oplus f_{20,35}^{(3)}=c_{20,45}^{(3)}\end{array}$ \\
\hline 19 & $\begin{array}{l}a_{19,59}^{(1)}=b_{19,59}^{(1)} \oplus 1, c_{19,9}^{(1)}=a_{20,9}^{(1)}, c_{19,59}^{(1)}=d_{19,59}^{(1)} \oplus 1, \\
e_{19,59}^{(1)}=f_{19,59}^{(1)} \oplus 1, g_{20,59}^{(1)}=h_{20,59}^{(1)} \oplus 1, f_{18,9}^{(1)}=c_{19,9}^{(1)}, \\
f_{18,59}^{(1)}=c_{18,59}^{(1)}, h_{18,59}^{(1)}=e_{18,59}^{(1)}\end{array}$ & $\left\{\begin{array}{l}a_{19,59}^{(3)}=b_{19,59}^{(3)} \oplus 1, c_{19,9}^{(3)}=a_{20,9}^{(3)}, c_{19,59}^{(3)}=d_{19,59}^{(3)} \oplus 1, \\
e_{19,59}^{(3)}=f_{19,59}^{(3)} \oplus, g_{20,59}^{(3)}=h_{20,59}^{(3)} \oplus 1, f_{18,9}^{(3)}=c_{19,9}^{(3)}, \\
f_{13,59}^{(3)}=c_{18,59}^{(3)}, h_{18,59}^{(3)}=e_{18,59}^{(3)}\end{array}\right.$ \\
\hline 18 & $a_{18,59}^{(1)}=g_{19,59}^{(1)}, c_{18,59}^{(1)}=a_{19,59}^{(1)}, f_{17,59}^{(1)}=c_{18,59}^{(1)}$ & $a_{18,59}^{(3)}=g_{19,59}^{(3)}, c_{18,59}^{(3)}=a_{19,59}^{(3)}, f_{17,59}^{(3)}=c_{18,59}^{(3)}$ \\
\hline 17 & $c_{17,59}^{(1)}=a_{18,59}^{(1)}, k_{8,59}=c_{17,59}^{(1)}$ & $c_{17,59}^{(3)}=a_{18,59}^{(3)}, k_{8,59}=c_{17,59}^{(3)}$ \\
\hline & s for $D_{3}$ & $\mathrm{~s}$ for $D_{4}$ \\
\hline 20 & $\begin{array}{l}b_{20,15}^{(1)}=a_{20,15}^{(1)} \oplus 1, d_{20,20}^{(1)}=c_{20,20}^{(1)} \oplus 1, d_{20,46}^{(1)}= \\
c_{20,46}^{(1)} \oplus 1, f_{20,10}^{(1)}=e_{20,10}^{(1)} \oplus 1, f_{20,30}^{(1)}=e_{20,30}^{(1)} \oplus 1, \\
h_{20,20}^{(1)}=g_{20,20}^{(1)} \oplus 1\end{array}$ & $\left\{\begin{array}{l}b_{20,15}^{(2)}=a_{20,15}^{(2)} \oplus 1, d_{20}^{(2)} \\
c_{20,46}^{(2)} \oplus 1, f_{20,10}^{(2)}=e_{20,1}^{(2)} \\
h_{20,20}^{(2)}=g_{20,20}^{(2)} \oplus 1\end{array}\right.$ \\
\hline & $\begin{array}{l}\hat{b}_{20,15}^{(1)}=b_{20,15}^{(1)}, \hat{b}_{20,37}^{(1)}=b_{20,37}^{(1)}, \hat{b}_{20,54}^{(1)}=b_{20,54}^{(1)}, \\
\hat{d}_{20,20}^{(1)}=d_{20,20}^{(1)}, \hat{d}_{20,46}^{(1)}=d_{20,46}^{(1)}, \hat{f}_{20,10}^{(1)}=f_{20,10}^{(1)}, \\
\hat{f}_{20,30}^{(1)}=f_{20,30}^{(1)}, \hat{h}_{20,40}^{(1)}=h_{20,40}^{(1)}\end{array}$ & $\left\{\begin{array}{l}\hat{b}_{20,15}^{(2)}=b_{20,15}^{(2)}, \hat{b}_{20,37}^{(2)}=b_{20,37}^{(2)}, \hat{b}_{20,54}^{(2)}=b_{20,5}^{(2)} \\
\hat{d}_{20,20}^{(2)}=d_{20,20}^{(2)}, \hat{d}_{20,46}^{(2)}=d_{20,46}^{(2)}, \hat{f}_{20,10}^{(2)}=f_{20,1}^{(2)} \\
\hat{f}_{20,30}^{(2)}=f_{20,30}^{(2)}, \hat{h}_{20,40}^{(2)}=h_{20,40}^{(2)}\end{array}\right.$ \\
\hline 21 & $\begin{array}{l}a_{21,37}^{(1)}=b_{20,37}^{(1)}, a_{21,50}^{(1)}=b_{20}^{(1)}, e_{21}^{(1)}, g_{20,44}^{(1)}, \\
g_{21,12}^{(1)}=a_{20,12}^{(1)}, g_{21,29}^{(1)}=a_{20,29}^{(1)}, g_{21,37}^{(1)}=b_{20,37}^{(1)}, \\
g_{21,54}^{(1)}=b_{20,54}^{(1)}, b_{21,37}^{(1)}=a_{21,37}^{(1)}+1, f_{21,44}^{(1)}=e_{21,44}^{(1)} \oplus \\
1, h_{21,12}^{(1)}=g_{21,12}^{(1)}+1, h_{21,37}^{(1)}=g_{21,37}^{(1)} \oplus 1\end{array}$ & $\left\{\begin{array}{l}a_{21,37}^{(2)}=b_{20,37}^{(2)}, a_{21,50}^{(2)}=b_{20,50}^{(2)}, e_{21,44}^{(2)}=g_{20,4}^{(2)} \\
g_{21,12}^{(2)}=a_{20,12}^{(2)}, g_{21,29}^{(2)}=a_{20,29}^{(2)}, g_{21,37}^{(2)}=b_{20,3}^{(2)} \\
g_{21,54}^{(2)}=b_{20,54}^{(2)}, b_{21,37}^{(2)}=a_{21,37}^{(2)}+1, f_{21,44}^{(2)}=e_{21,44}^{(2)} \\
1, h_{21,12}^{(2)}=g_{21,12}^{(2)}+1, h_{21,37}^{(2)}=g_{21,37}^{(2)} \oplus 1\end{array}\right.$ \\
\hline 22 & $\begin{array}{l}e_{22,29}^{(1)}=g_{22,29}^{(1)}, e_{22,54}^{(1)}=g_{22,54}^{(1)}, f_{22,54}^{(1)}=e_{22,54}^{(1)} \oplus 1, \\
g_{22,50}^{(1)}=a_{21,50}^{(1)}, h_{22,50}^{(1)}=g_{22,50}^{(1)}\end{array}$ & $\begin{array}{l}e_{22,29}^{(2)}=g_{22,29}^{(2)}, e_{22,54}^{(2)}=g_{22,54}^{(2)}, f_{22,54}^{(2)}= \\
g_{22,50}^{(2)}=a_{21,50}^{(2)}, h_{22,50}^{(2)}=g_{22,50}^{(2)}\end{array}$ \\
\hline 23 & $c_{23,29}^{(1)}=e_{22,29}^{(1)}, d_{23,29}^{(1)}=c_{23,29}^{(1)} \oplus 1$ & $c_{23,29}^{(2)}=e_{22,29}^{(2)}, d_{23,29}^{(2)}=c_{23,29}^{(2)} \oplus 1$ \\
\hline
\end{tabular}

Table 5. The conditions for Differential Path $D_{2}$ which hold with probability $2^{-7.4}$.

\begin{tabular}{|c|c|c|}
\hline round & conditions & $\mathrm{pr}$ \\
\hline 20 & $\begin{array}{l}a_{20,9}^{(3)}=a_{20,9}^{(1)}, a_{20}^{(3)}=a_{20,34}^{(1)}, b_{20,39}^{(3)}=b_{20,39}^{(1)}, c_{20,9}^{(3)}=c_{20,9}^{(1)}, c_{20,31}^{(3)}=c_{20,31}^{(1)}, \\
c_{20,45}^{(3)}=c_{20,45}^{(1)}, d_{20,51}^{(3)}=d_{20,51}^{(1)}, f_{20,9}^{(3)}=f_{20,9}^{(1)}, f_{20,21}^{(3)}=f_{20,21}^{(1)}, f_{20,31}^{(3)}=f_{20,31}^{(1)}, \\
f_{20,35}^{(3)}=f_{20,35}^{(1)}, f_{20,45}^{(3)}=f_{20,45}^{(1)}, f_{20,49}^{(3)}=f_{20,49}^{(1)}, f_{20,63}^{(3)}=f_{20,63}^{(1)}, g_{20,59}^{(3)}=g_{20,9}^{(1)}, \\
h_{20,4}^{(3)}=h_{20,4}^{(1)}, h_{20,9}^{(3)}=h_{20,9}^{(1)}, h_{20,34}^{(3)}=h_{20,34}^{(1)}, f_{20,43}^{(3)}=f_{20,43}^{(1)}\end{array}$ & 1 \\
\hline 19 & $\begin{array}{l}b_{19,59}^{(3)}=b_{19,59}^{(1)}, a_{19,59}^{(3)}=a_{19,59}^{(1)}(0.75), d_{19,34}^{(3)}=d_{19,34}^{(1)}, d_{19,59}^{(3)}=d_{19,59}^{(1)}, \\
c_{19,9}^{(3)}=c_{19,9}^{(1)}(0.87), c_{19,59}^{(3)}=c_{19,59}^{(1)}(0.94), f_{19,9}^{(3)}=f_{19,9}^{(1)}, f_{19,31}^{(3)}=f_{19,31}^{(1)}, \\
f_{19,45}^{(3)}=f_{19,45}^{(1)}, f_{19,59}^{(3)}=f_{19,59}^{(1)}, e_{19,59}^{(3)}=e_{19,59}^{(1)}(0.875), h_{19,59}^{(3)}=h_{19,59}^{(1)}, \\
g_{19,59}^{(3)}=g_{19,59}^{(1)}(0.97)\end{array}$ & 0.52 \\
\hline 18 & $\begin{array}{l}a_{18,59}^{(3)}=a_{18,59}^{(1)}(0.687), c_{18,59}^{(3)}=c_{18,59}^{(1)}(0.29), f_{18,9}^{(3)}=f_{18,9}^{(1)}, f_{18,59}^{(3)}= \\
f_{18,59}^{(1)}(0.25), h_{18,59}^{(3)}=h_{18,59}^{(1)}(0.937)\end{array}$ & 0.047 \\
\hline 17 & $c_{17,59}^{(3)}=c_{17,59}^{(1)}(0.5), f_{17,59}^{(3)}=f_{17,59}^{(1)}(0.5)$ & 0.25 \\
\hline
\end{tabular}


Table 6. The conditions for Differential Path $D_{4}$ which hold with probability $2^{-8.4}$.

\begin{tabular}{|c|c|c|}
\hline round & conditions & $\mathrm{pr}$ \\
\hline 20 & $\begin{array}{l}a_{20,12}^{(2)}=a_{20,12}^{(1)}, a_{20,15}^{(2)}=a_{20,15}^{(1)}, a_{20,29}^{(2)}=a_{20,29}^{(1)}, b_{20,15}^{(2)}=b_{20,15}^{(1)}, b_{20,37}^{(2)}= \\
b_{20,37}^{(1)}, b_{20,54}^{(2)}=b_{20,54}^{(1)}, c_{20,20}^{(2)}=c_{20}^{(1)}, c_{20,37}^{(2)}=c_{20,37}^{(1)}, c_{20}^{(2)}, 46=c_{20,46}^{(1)}, \\
c_{20,50}^{(2)}=c_{20,50}^{(1)}, d_{20,20}^{(2)}=d_{20,20}^{(1)}, d_{20,46}^{(2)}=d_{20,46}^{(1)}, e_{20,10}^{(2)}=e_{20,10}^{(1)}, e_{20,30}^{(2)}= \\
e_{20,30}^{(1)}, f_{20,10}^{(2)}=f_{20,10}^{(1)}, f_{20,30}^{(2)}=f_{20,30}^{(1)}, g_{20,20}^{(2)}=g_{20,20}^{(1)}, g_{20,40}^{(2)}=g_{20,40}^{(1)} \\
g_{20,44}^{(2)}=g_{20,44}^{(1)}, h_{20,20}^{(2)}=h_{20,20}^{(1)}, h_{20,40}^{(2)}=h_{20,40}^{(1)}\end{array}$ & 1 \\
\hline & $\begin{array}{l}\hat{b}_{20,15}^{(2)}=\hat{b}_{20,15}^{(1)}, \hat{b}_{20,37}^{(2)}=\hat{b}_{20,37}^{(1)}, \hat{b}_{20,54}^{(2)}=\hat{b}_{20,54}^{(1)}, \hat{d}_{20,20}^{(2)}=\hat{d}_{20,20}^{(1)}, \hat{d}_{20,46}^{(2)}= \\
\hat{d}_{20,46}^{(1)}, \hat{f}_{20,10}^{(2)}=\hat{f}_{20,10}^{(1)}(0.5), \hat{f}_{20,30}^{(2)}=\hat{f}_{20,30}^{(1)}, \hat{h}_{20,40}^{(2)}=\hat{h}_{20,40}^{(1)}\end{array}$ & 0.5 \\
\hline 21 & $\begin{array}{l}a_{21,37}^{(2)}=a_{21,37}^{(1)}, a_{21,50}^{(2)}=a_{21,50}^{(1)}(0.97), b_{21,37}^{(2)}=b_{21,37}^{(1)}, e_{21,44}^{(2)}=e_{21,44}^{(1)}(0.5), \\
f_{21,44}^{(2)}=f_{22,44}^{(1)}, g_{21,12}^{(2)}=g_{21,12}^{(1)}(0.875), g_{21,29}^{(2)}=g_{21,29}^{(1)}, g_{21,37}^{(2)}=g_{21,37}^{(1)}(0.875), \\
g_{21,54}^{(2)}=g_{21,54}^{(1)}, h_{21,12}^{(2)}=h_{21,12}^{(1)}(0.875), h_{21,37}^{(2)}=h_{21,37}^{(1)}\end{array}$ & 0.32 \\
\hline 22 & $\begin{array}{l}e_{22,29}^{(2)}=e_{22,29}^{(1)}(0.84), e_{22,54}^{(2)}=e_{22,54}^{(1)}(0.75), f_{22,54}^{(2)}=f_{22,54}^{(1)}(0.5), g_{22,50}^{(2)}= \\
g_{22,50}^{(1)}(0.97), h_{22,50}^{(2)}=h_{22,50}^{(1)}(0.5)\end{array}$ & 0.15 \\
\hline 23 & $c_{23,29}^{(2)}=c_{23,29}^{(1)}(0.24), d_{23,29}^{(2)}=d_{23,29}^{(1)}(0.5)$ & 0.12 \\
\hline
\end{tabular}

- The complexity of 34-round distinguisher (rounds $3-37$ ) is about $2^{2 \cdot(37+6)} \times 3^{35+13+18} \approx 2^{190.6}$.

- The complexity of 35-round distinguisher (rounds $3-38$ ) is about $2^{2 \cdot(72+82)}=2^{308}$.

- The complexity of 36 -round distinguisher (rounds $2-38$ ) is about $2^{2 \cdot(72+82+73)}=2^{454}$.

Remark: For the 32-/33-/34-round attacks, we use the Type III boomerang distinguisher, the complexity for the best algorithm is $2^{256}$; for the 35 -/36-round attacks, we use the Type I boomerang distinguisher, the generic complexity is about $2^{512}$. Note that the initial and final key-additions are included in our 32-round reduced Skein-512; they are not included in the distinguishers for 33 to 36 rounds.

In the following, we give examples of the quartets to show that our technique used for 32 to 36 rounds attack is valid. Table 7 gives a zero-sum quartet for rounds 5-32 of Skein-512 (the initial and final subkeys are not included) with $\oplus_{i=1}^{4} V_{5}^{(i)}=0$ and $\oplus_{i=1}^{4} V_{36}^{(i)}=0$. The complexity of attack is about $2^{32}$. Table 8 gives a zero-sum quartet for rounds 8-36 of Skein-512 with $\oplus_{i=1}^{4} V_{8}^{(i)}=0$ and $\oplus_{i=1}^{4} \hat{V}_{36}^{(i)}=0$ (the initial and final subkeys are included). The complexity of attack is about $2^{40.5}$.

\subsection{The incompatible for the boomerang attack on Threefish-512 with the old rotation constants}

At Asiacrypt 2009, Aumasson et al. first presented the boomerang distinguishers on Threefish512 reduced to 35 rounds. We studied the differential paths used to boomerang attack in Tables 6 and 7 of [2], and found that they used an inverse permutation instead of the original one. We correct the permutation and give the middle 8-round differential paths (see Table 9) using the key differences proposed in [1] under the old rotation constants. For the top path, the MSB differences are set in $k_{7}$ and $t_{0}$; for the bottom path, the MSB differences are set in $k_{2}, k_{3}, t_{0}$ and $t_{1}$.

From the bottom path, it is easy to know that $\hat{d}_{16,11}^{(1)}=\hat{c}_{16,11}^{(1)} \oplus 1, \hat{d}_{16,11}^{(2)}=\hat{c}_{16,11}^{(2)} \oplus 1$. From the top path, we know that $\hat{d}_{16,11}^{(1)}=\hat{d}_{16,11}^{(2)}$, so we get $\hat{c}_{16,11}^{(1)}=\hat{c}_{16,11}^{(2)}$. But from the top differential, it's obvious that $\hat{c}_{16,11}^{(1)}=\hat{c}_{16,11}^{(2)} \oplus 1$. Hence a contradiction generates. Similarly, the differences on bit 41 for the top and bottom paths are also incompatible. 
Table 7. A quartet that satisfies the paths for rounds 5-36.

\begin{tabular}{|c|c|}
\hline \multicolumn{2}{|r|}{ Message of Round 5} \\
\hline$M^{(1)}$ & $\begin{array}{l}\text { efeffeca89966f57 b9ede50911910872 b80346f52e40f9b2 413a42e591e3d564 } \\
\text { b854665ac709fdc1 5b81218db8689f63 1454025d1e252a79 40086ca8b43d3382 }\end{array}$ \\
\hline$M^{(2)}$ & $\begin{array}{l}\text { efefeecb09966f57 b9ede50891910872 b40346f52e40f9b2 453a42e591e3d564 } \\
\text { b854661ac709fdc1 5b8121cdb8689f63 1455025d9ea52a39 40086ca8343d33c2 }\end{array}$ \\
\hline$M^{(3)}$ & $\begin{array}{l}\text { 5b44c68c6c74d8d8 462dcb0d8f65c514 4660e299d27ed556 1622a67e6860f1b3 } \\
8631 \mathrm{f} 78 \mathrm{ea} 11186 \mathrm{~d} 9 \text { 29bf5dee4c4708bf 54cb280ae171a9fd df5814e7668fdf95 }\end{array}$ \\
\hline$M^{(4)}$ & $\begin{array}{l}\text { 5b44d68dec74d8d8 462dcb0c0f65c514 4a60e299d27ed556 1222a67e6860f1b3 } \\
8631 f 7 c e a 11186 d 9 \text { 29bf5dae4c4708bf 54ca280a61f1a9bd df5814e7e68fdfd5 }\end{array}$ \\
\hline \multicolumn{2}{|r|}{ Key } \\
\hline$K^{(1)}$ & $\begin{array}{l}\text { fd4707e3dc7b1c35 3f64c6f0bd13466a 45e7c90173366b70 dc71a6f93dbfc9d5 } \\
5 c 977 a 7 b b c 2 d b e 6 d \text { 56889bd71af7189f 8bc7bcb9d86167a1 0091f15b4d1aeaee }\end{array}$ \\
\hline$K^{(2)}$ & $\begin{array}{l}\text { f94707e3dc7b1c35 3f64c6f0bd13466a 45e7c90173366b70 dc71a6f93dbfc9d5 } \\
\text { 5c977a7bbc2dbe6d 56889bd71af7189f 8bc7bcb9d86167a1 0091f15b4d1aeaee }\end{array}$ \\
\hline$K^{(3)}$ & $\begin{array}{l}\text { fd4707e3dc7b1c35 3f64c6f0bd13466a 45e7c90173366b70 5c71a6f93dbfc9d5 } \\
\text { dc977a7bbc2dbe6d 56889bd71af7189f 8bc7bcb9d86167a1 0091f15b4d1aeaee }\end{array}$ \\
\hline$K^{(4)}$ & $\begin{array}{l}\text { f94707e3dc7b1c35 3f64c6f0bd13466a 45e7c90173366b70 5c71a6f93dbfc9d5 } \\
\text { dc977a7bbc2dbe6d 56889bd71af7189f 8bc7bcb9d86167a1 0091f15b4d1aeaee }\end{array}$ \\
\hline \multicolumn{2}{|r|}{ Tweak } \\
\hline $\begin{array}{l}(1) \\
{ }^{(3)}, T^{(2)} \\
T^{(4)}\end{array}$ & $\begin{array}{l}\text { 55422f07b9ea59be 511ad7aa13272cc9 51422f07b9ea59be 551ad7aa13272cc9 } \\
55422 \text { f07b9ea59be d11ad7aa13272cc9 51422f07b9ea59be d51ad7aa13272cc9 }\end{array}$ \\
\hline
\end{tabular}

Table 8. A quartet that satisfies the paths for rounds 8-36 including the first and final subkeys.

\begin{tabular}{|c|c|}
\hline \multicolumn{2}{|r|}{ Message of Round 8} \\
\hline \multirow{2}{*}{$M^{(1)}$} & 81eb65560efb565c 42171413b9dae252 ba7f35e83ceec8b7 d5dbcf318a0ecf74 \\
\hline & $5 \mathrm{~d} 1 \mathrm{c} 176606 \mathrm{c51b} 45$ 4f8fc8fc188100d4 45d34efc985185f5 673059aaf448427c \\
\hline \multirow{2}{*}{$M^{(2)}$} & 81eb65560efb565c 42171413b9dae252 ba7f35e83ceec8b7 d5dbcf318a0ecf74 \\
\hline & $5 \mathrm{~d} 1 \mathrm{c} 176606 \mathrm{c} 51 \mathrm{~b} 45$ 4f8fc8fc188100d4 45d34efc985185f5 6b3059aaf448427c \\
\hline \multirow{2}{*}{$M^{(3)}$} & f96c2ea16f7aa900 7dbe4b7cc9bef8ea f94e7e6cff763332 f44decb0fcb6ecac \\
\hline & $7 f 30973 f a d 83191 f$ 94591dff30d2e161 74c7323813fc5c42 54e6ccf74a6a1d11 \\
\hline \multirow{2}{*}{$M^{(4)}$} & f96c2ea16f7aa900 7dbe4b7cc9bef8ea f94e7e6cff763332 f44decb0f cb6ecac \\
\hline & $7 f 30973 f a d 83191 f$ 94591dff30d2e161 74c7323813fc5c42 58e6ccf74a6a1d11 \\
\hline \multicolumn{2}{|r|}{ Key } \\
\hline$K^{(1)}$ & $\begin{array}{l}\text { bf 07320940fa73f1 64561111c05cc195 bbf500154032fa6d 8dff001fb0239bbf } \\
\text { 5e36a0172124dd89 50e99cdbc81bab42 3ac1c8825115600a 12b40efea4188dab }\end{array}$ \\
\hline \multirow{2}{*}{$K^{(2)}$} & bb07320940fa73f1 64561111c05cc195 bbf500154032fa6d 8dff001fb0239bbf \\
\hline & 5e36a0172124dd89 50e99cdbc81bab42 3ac1c8825115600a 12b40efea4188dab \\
\hline$K^{(3)}$ & $\begin{array}{l}\text { bf 07320940fa73f1 64561111c05cc195 bbf500154032fa6d 0dff001fb0239bbf } \\
\text { de36a0172124dd89 50e99cdbc81bab42 3ac1c8825115600a 12b40efea4188dab }\end{array}$ \\
\hline \multirow[t]{2}{*}{$K^{(4)}$} & $\begin{array}{l}\text { bb07320940fa73f1 64561111c05cc195 bbf500154032fa6d 0dff001fb0239bbf } \\
\text { de36a0172124dd89 50e99cdbc81bab42 3ac1c8825115600a 12b40efea4188dab }\end{array}$ \\
\hline & Tweak \\
\hline \multirow{2}{*}{$\begin{array}{l}T^{(1)}, T^{(2)} \\
T^{(3)}, T^{(4)}\end{array}$} & 8fe4eab7841221ae 82aeedc8d61e677b 8be4eab7841221ae 86aeedc8d61€ \\
\hline & 8fe4eab7841221ae 02aeedc8d61e677b 8be4eab7841221ae 06aeedc8d61e677b \\
\hline
\end{tabular}


Table 9. The modified differential path in the middle rounds used for boomerang attacks of Skein-512 in [1].

\begin{tabular}{|c|c|c|}
\hline Rd & shifts & The Difference for the top path from rounds $12-16$ \\
\hline \multirow{2}{*}{$K_{3}$} & & 0000000000000000000000000000000000000000000000000000000000000000 \\
\hline & & 8000000000000000000000000000000000000000000000000000000000000000 \\
\hline \multirow{2}{*}{12} & 33,49 & 0000000000000000000000000000000000000000000000000000000000000000 \\
\hline & 8,42 & 8000000000000000000000000000000000000000000000000000000000000000 \\
\hline \multirow{2}{*}{13} & 39,27 & 0000000000000000000000000000000080000000000000000000000000000000 \\
\hline & 41,14 & 0000000000000000800000000000000000000000000000000000000000000000 \\
\hline \multirow{2}{*}{14} & 29,26 & 8000000000000000000000000000000080000000000000000000000000000000 \\
\hline & 11,9 & 0000000000000000800001000000000000000000000000008000000000000000 \\
\hline \multirow{2}{*}{15} & 33,51 & 8000000000000000800000000000000080000100000000008000000000000100 \\
\hline & 39,35 & 800000000000000080080100000004008000000000000000800000 \\
\hline \multirow{2}{*}{16} & & 00000100000001000000000100000000000801000000040000000 \\
\hline & & $0000000000000000000 \mathrm{a} 01400400840000000000000000000004010000000100$ \\
\hline \multirow{2}{*}{$K_{4}$} & & 000000000000000000000000000000000000000000000000800000000 \\
\hline & & 80000000000000000000000000000000000000000000000080000 \\
\hline \multirow{2}{*}{16} & & 0000010000000100000000010000000000080100000004008000000400000000 \\
\hline & & $8000000000000000000 a 01400400840000000000000000000804010000000100$ \\
\hline & & The Difference for the bottom path from rounds $16-20$ \\
\hline \multirow{2}{*}{16} & 38,30 & 40084010801020244000400080002004044001800100040004400080 \\
\hline & 50,53 & 0000000000040090000000000004008002000000000080100000000 \\
\hline \multirow{2}{*}{17} & 48,20 & 0000010001000000000001000000000000000000000000100000000000000010 \\
\hline & 43,31 & 0200000000000000020000000000000000080010001000200000000000100020 \\
\hline \multirow{2}{*}{18} & 34,14 & 0000000000000000000000000000000000000000000000000000000000000000 \\
\hline & 15,27 & 0008001000000000000000100000000000000000010000000000000001000000 \\
\hline \multirow{2}{*}{19} & 26,12 & 0000000000000000000000000000000000080000000000000008000000000000 \\
\hline & 58,7 & 0000000000000000000000000000000000000000000000000000000000000000 \\
\hline \multirow{2}{*}{20} & & 0000000000000000000000000000000000000000000000000000000000000000 \\
\hline & & 0000000000000000000000000000000000000000000000008000000000000000 \\
\hline \multirow{2}{*}{$K_{5}$} & & 0000000000000000000000000000000000000000000000000000000000000000 \\
\hline & & 0000000000000000000000000000000000000000000000008000000000000000 \\
\hline
\end{tabular}




\section{$5 \quad$ Key Recovery Attack on Reduced Threefish-512}

Our boomerang distinguishers for 32 to 34 rounds Skein-512 are also applicable to (related) key recovery attack on Threefish-512. In this case, the complexity for the middle 8 rounds are added, and the initial and final subkeys are included. For the fixed input and output differences $\alpha$ and $\gamma$, the probabilities of the boomerang distinguishers for Threefish-512 reduced to 32 (rounds 4-36), 33 (rounds 4-37), 34 (rounds $3-37$ ) rounds are $2^{-177}, 2^{-301}$ and $2^{-419}$ respectively.

Consequently, we can mount key recovery attacks on reduced Threefish-512 for $1 / 4$ of the key space, with complexities $2^{181}, 2^{305}$ and $2^{424}$, respectively. We give the procedure of the key recovery attack on 32-round Threefish-512 as an example.

1. For $i=1, \ldots, 2^{179}$

(a) Randomly choose plaintext $P_{1}^{i}$, compute $P_{2}^{i}=P_{1}^{i} \oplus \alpha$.

(b) Encrypt plaintext pair $\left(P_{1}^{i}, P_{2}^{i}\right)$ with $K^{(1)}, K^{(2)}$ respectively to get $\left(C_{1}^{i}, C_{2}^{i}\right)$. Compute $C_{3}^{i}=C_{1}^{i} \oplus \delta, C_{4}^{i}=C_{2}^{i} \oplus \delta$. Then decrypt $\left(C_{3}^{i}, C_{4}^{i}\right)$ with $K^{(3)}, K^{(4)}$ respectively to get $\left(P_{3}^{i}, P_{4}^{i}\right)$.

(c) Check whether $P_{3}^{i} \oplus P_{4}^{i}=\alpha$, if so, store the quartet $\left(C_{1}^{i}, C_{2}^{i}, C_{3}^{i}, C_{4}^{i}\right)$.

2. (a) Guess 128 bits of the final subkey words $K_{9, a}, K_{9, b}$ and subtract them with the corresponding words of each element of quartets stored in Step 1. If for all the quartets, whose resulting words satisfy that the XOR differences before the key addition, we store this 128-bit subkey pair $\left(K_{9, a}, K_{9, b}\right)$.

(b) Similarly, sequently guess $\left(K_{9, c}, K_{9, d}\right)$ and $\left(K_{9, f}, K_{9, h}\right)$ and check whether the required conditions are satisfied. If yes, store the corresponding key words.

3. Search the remaining 128 bits of the final subkey by brute force.

The complexity is dominated by Step 1 , which is about $2^{181} 32$-round encryptions. The expected number of quartets passed Step 2(a) for a false key is $4 \times 2^{-6}=2^{-4}$. Let $Y$ be the number of the quartets passed Step 2(a) for a false key, using the Poisson distribution, we have $\operatorname{Pr}(Y \geq 4) \approx 0$. The expected quartets passed Step 2(a) for the right key is 4 . Let $Z$ be the number of the quartets passed Step 2 (a) for the right key, $\operatorname{Pr}(Z \geq 4) \approx 0.9$. The success rate of Step 2 (b) is similar.

\section{Conclusions}

In this paper, we apply the boomerang attack to distinguish the compression function of Skein512 reduced to 36 (out of 72) rounds from a random function. We use the key difference in bit 59 instead of the difference in the most significant bit to avoid the contradiction in the previous attack for boomerang attacks on Threefish-512. We also reveal that the differential paths used in the boomerang distinguisher in the middle rounds are not independent. Our boomerang distinguishers are applicable to the key recovery attack for Threefish-512 reduced to 34 rounds. Future works on Skein-512 might apply the rebound attack [8] to Threefish, although it looks very difficult to combine two short differential paths to a long one.

\section{References}

1. J.-P. Aumasson, C. Calik, W. Meier, O. Ozen, R.C.W.Phan, K. Varici: Improved Cryptanalysis of Skein. In: Matsui, M. (ed.) ASIACRYPT 2009. LNCS, vol. 5912, pp. 542-559. Springer, Heidelberg (2009)

2. J.Aumasson1 et. al, Improved Cryptanalysis of Skeinhttp://eprint.iacr.org/2009/438.pdf

3. E.Biham, O.Dunkelman, N.Keller, The Rectangle Attack - Rectangling the Serpent. In: Pfitzmann, B. (Eds.) EUROCRYPT 2001. LNCS, vol. 2045, pp. 340-357. Springer, Heidelberg (2001)

4. E.Biham, O.Dunkelman, N.Keller, Related-Key Boomerang and Rectangle Attacks. In: Cramer, R. (ed.) EUROCRYPT 2005. LNCS, vol. 3494, pp. 507-525. Springer, Heidelberg (2005) 
5. A.Biryukov, M.Lamberger, F.Mendel, I.Nikolic, Second-Order Differential Collisions for Reduced SHA-256. In D.H.Lee and X.Wang (Eds.): Asiacrypt 2011, LNCS 7073, pp.270-287. Springer, Heidelberg (2011)

6. J.Z.Chen, K.T.Jia, Improved Related-Key Boomerang Attacks on Round-Reduced Threefish-512. In J. Kwak et al. (Eds.): ISPEC 2010, LNCS 6047, pp. 1-18, Springer, Heidelberg (2010).

7. N. Ferguson, S. Lucks, B. Schneier, D. Whiting, M. Bellare, T. Kohno, J. Callas, J. Walker: The Skein Hash Function Family, http://www.schneier.com/skein1.3.pdf

8. F. Mendel, C. Rechberger, M. Schläffer, Søren S. Thomsen: The Rebound Attack: Cryptanalysis of Reduced Whirlpool and Grøstl. In: O. Dunkelman (ed.) FSE 2009. LNCS, vol. 5665, pp. 260-276. Springer, Heidelberg (2009)

9. F. Mendel, C. Rechberger, M. Schläffer, S. Thomsen, Rebound Attack on the Reduced Grøstl Hash Function. In J.Pieprzyk (Ed.): CT-RSA 2010, LNCS 5985, pp.350-365. Springer, Heidelberg (2010)

10. D. Khovratovich, I. Nikolić, Rotational Cryptanalysis of ARX. In S.Hong and T. Iwata (Ed.): FSE 2010, LNCS 6147, pp.333-346. Springer, Heidelberg (2010)

11. D. Khovratovich, I. Nikolić, C. Rechberger: Rotational Rebound Attacks on Reduced Skein. In: M. Abe (Ed.): ASIACRYPT 2010, LNCS 6477, pp. 1-19. Springer, Heidelberg (2010)

12. D. Khovratovich, C. Rechberger and A. Savelieva, Bicliques for Preimages: Attacks on Skein-512 and the SHA-2 family. FSE 2012.

13. J.Kelsey, T.Khono, B.Schneier, Amplified Boomerang Attacks Against Reduced-Round MARS and Serpent. In: Schneier, B. (ed.) FSE 2000. LNCS 1978, pp.75-93. Springer, Heidelberg (2001)

14. G.Leurent, A.Roy, Boomerang Attacks on Hash Function Using Auxiliary Differentials. In O.Dunkelman (ed.), CT-RSA 2012. LNCS 7178, pp.215-230. Springer, Heidelberg (2012).

15. B.Z. Su, W.L. Wu, S. Wu, and L. Dong: Near-Collisions on the Reduced-Round Compression Functions of Skein and BLAKE. In: S.-H. Heng, R.N. Wright, and B.-M. Goi (ed.): CANS 2010, LNCS 6467, pp. 124-139. Springer, Heidelberg (2010)

16. D.Wagner, The Boomerang Attack. In: L.R.Knudsen(ed.) FSE 1999. LNCS 1636, pp. 156-170. Springer, Heidelberg (1999)

17. D.Wagner, A Generalized Birthday Problem. In: M.Yung(ed.) Crypto 2002, LNCS 2442, pp.288-303. Springer, Heidelberg $(2002)$

18. X.Y. Wang, H.B. Yu: How to Break MD5 and Other Hash Functions. In: Cramer, R. (ed.) EUROCRYPT 2005. LNCS 3494, pp. 19-35. Springer, Heidelberg (2005)

19. X.Y. Wang, Y. L. Yin, H.B.Yu: Finding Collisions in the Full SHA-1. In: Shoup, V. (ed.) CRYPTO 2005. LNCS 3621, pp. 17-36. Springer, Heidelberg (2005)

20. H.B.Yu, J.Z.Chen, K.T.Jia, X.Y.Wang, Near-Collision Attack on the Step-Reduced compression Function of Skein-256. Cryptology ePrint Archive, Report 2011/148(2011),http://eprint.iacr.org 
Table 10. The top differential path used for boomerang attacks of Skein-512.

\begin{tabular}{|c|c|c|c|}
\hline $\mathrm{Rd}$ & Shifts & Difference & $\operatorname{Pr}$ \\
\hline \multirow{2}{*}{2} & 17,49 & 0c030025814280b4 08020024800290a0 84689060080a4234 80209020280a0224 & \multirow{2}{*}{$2^{-73}$} \\
\hline & 36,39 & $603 a 002310842201403800231204602009421184 \mathrm{e} 3408 \mathrm{c} 32 \quad 906008062408 \mathrm{c} 22$ & \\
\hline \multirow{2}{*}{3} & 44,9 & 0448004020004010044800042000001020020000028042212002000002004021 & \multirow{2}{*}{$2^{-35}$} \\
\hline & 54,56 & 0044110481000010004402040100401004010001014010140001000100401004 & \\
\hline \multirow{2}{*}{4} & & 0000000000800240000100008000004000001100800040000000010000004000 & \multirow{2}{*}{$2^{-24}$} \\
\hline & & 0400000001000010040000000100001000000044000040000400000400004000 & \\
\hline \multirow{2}{*}{$K_{1}$} & & 0000000000000000000000000000000000000000000000000000000000000000 & \\
\hline & & 0000000000000000040000000000000000000000000000000400000000000000 & \\
\hline \multirow{2}{*}{4} & 39,30 & 0001000080000040 & \multirow{2}{*}{1} \\
\hline & 34,24 & 0000000001000010 & \\
\hline \multirow{2}{*}{5} & 13,50 & 0000100080000000000000008000000004000000000000000400000000000000 & \multirow{2}{*}{$2^{-8}$} \\
\hline & 10,17 & 000000400000000000000040000000000001000080800040000 & \\
\hline \multirow{2}{*}{6} & 25,29 & 0000000000000000000000000000000000000000000000000000000000000000 & \multirow{2}{*}{$2^{-3}$} \\
\hline & 39,43 & 0001000000800000000100000000000000001000000000000000100000000000 & \\
\hline \multirow{2}{*}{7} & 8,35 & 0000000000000000000000000000000000000000008000000000000000 & \multirow{2}{*}{$2^{-1}$} \\
\hline & 56,22 & 000000000000000000000000000000000000000000 & \\
\hline \multirow{2}{*}{8} & & 0000000000000000000000000000000000000000000000000000000000000000 & \multirow{2}{*}{$2^{-1}$} \\
\hline & & 0000000000000000000000000000000000000000000000000400 & \\
\hline \multirow{3}{*}{$K_{2}$} & & 0000000000000000000000000000000000000000000 & \multirow{2}{*}{-} \\
\hline & & 0000000000000000000000000000000000000000000000000400000000000000 & \\
\hline & & no differences in rounds 9-16 & \\
\hline \multirow{2}{*}{$K_{4}$} & & 00000000000000000000000000000000000000000000000000 & \multirow[t]{2}{*}{-} \\
\hline & & 040000000000000000000000000000000000000000000000000 & \\
\hline \multirow{2}{*}{16} & 46,36 & 0000000000000000000000000000000000000000000000000000000000000000 & \multirow[b]{2}{*}{1} \\
\hline & 19,37 & $-\quad 000000000000000000000000000000000000000000000000$ & \\
\hline \multirow{2}{*}{17} & 33,27 & 0000000000000000000000000000000004000000000000000000000000000000 & \multirow{2}{*}{$2^{-2}$} \\
\hline & 14,42 & 0000000000000000040000000000000000000000000000000000000000000000 & \\
\hline \multirow{2}{*}{18} & 17,49 & 0400000000000000000000000000000004000000000000000000000000000000 & \multirow{2}{*}{$2^{-5}$} \\
\hline & 36,39 & 0000000000000000040000000000010000000000000000000400000000000000 & \\
\hline \multirow{2}{*}{19} & 44,9 & 0400000000000000040000000000000004000000000001000400000200000000 & \multirow{2}{*}{$2^{-9}$} \\
\hline & 54,56 & 0400000000000000040010004000010004000000000000000400000000000000 & \\
\hline 20 & & 0000000200000100000000400000000000001000400001000004000000000000 & \\
\hline & & 0000000000000000400110044010010000000000000000000000040200000108 & \\
\hline
\end{tabular}


Table 11. The bottom differential path used for boomerang attacks of Skein-512.

\begin{tabular}{|c|c|c|c|}
\hline $\mathrm{Rd}$ & hifts & Difference & $\operatorname{Pr}$ \\
\hline \multirow{2}{*}{20} & & 0000000010004800002000100000400000022010000800000000200000080000 & \multirow{2}{*}{$2^{-7}$} \\
\hline & & 8000000020000200800000002000020000000880000800008000008000080000 & \\
\hline \multirow{2}{*}{$K_{5}$} & & 0000000000000000000000000000000000000000000000000000000000000000 & \\
\hline & & 000000000000000800000000000000000000000000000008000000000000000 & \\
\hline \multirow{2}{*}{20} & 39,30 & 0020001000004000 & \multirow{2}{*}{$2^{-9}$} \\
\hline & 34,24 & $\begin{array}{llll}- & 0000000020000200 & - & 0000008000080000\end{array}$ & \\
\hline \multirow{2}{*}{21} & 13,50 & 0002001000000000000000100000000080000000000000008000000000000000 & \multirow{2}{*}{$2^{-7}$} \\
\hline & 10,17 & 0000080000000000000008000000000000200010100008000000001000000800 & \\
\hline \multirow{2}{*}{22} & 25,29 & 0000000000000000000000000000000000000000000000000000000000000000 & \multirow{2}{*}{$2^{-7}$} \\
\hline & 39,43 & 0020000010000000002000000000000000020000000000000002000000000000 & \\
\hline \multirow{2}{*}{23} & 8,35 & 00000000000000000000000000000000000000001000000000 & \multirow{2}{*}{$2^{-3}$} \\
\hline & 56,22 & 00000000000000000000000000000000000000000000000000 & \\
\hline \multirow{2}{*}{24} & & 000000000000000000000000000000000000000000000000000 & \multirow{2}{*}{$2^{-1}$} \\
\hline & & 00000000000000000000000000000000000000000000000080 & \\
\hline \multirow{3}{*}{$K_{6}$} & & 00000000000000000000000000000000000000000 & \\
\hline & & 000000000000000000000000000000000000000000 & \\
\hline & & no differences in Rounds 2 & \\
\hline \multirow{2}{*}{$K_{8}$} & & 00000000000000000000000000000000000000000000000000 & \multirow{2}{*}{-} \\
\hline & & 80000000000000000000000000000000000000000000000000 & \\
\hline \multirow{2}{*}{32} & 46,36 & 000000000000000000000000000000000000000000000 & \multirow{2}{*}{1} \\
\hline & 19,37 & 80000000000000000000000000000000000000000 & \\
\hline \multirow{2}{*}{33} & 33,27 & 00000000000000000000000000000000800000000000000000 & \multirow{2}{*}{1} \\
\hline & 14,42 & 00000000000000008000000000000000000000000000000000 & \\
\hline \multirow{2}{*}{34} & 17,49 & 80000000000000000000000000000000800000000 & \multirow{2}{*}{1} \\
\hline & 36,39 & 000000000000000080000000000020000000000000000000800 & \\
\hline \multirow{2}{*}{35} & 44,9 & 8000000000000000800000000000000080000000000020008000004000000000 & \multirow{2}{*}{$2^{-1}$} \\
\hline & 54,56 & 800000000000000080020008000020008000000000000000800 & \\
\hline \multirow{2}{*}{36} & & 000000400000200000000800000000000002000800002000008 & \multirow{2}{*}{$2^{-5}$} \\
\hline & & 00000000000000000022008802002008000000000000000000 & \\
\hline$K_{0}$ & & 0000000000000000000000000000000000000000000000008000000000000000 & \\
\hline 19 & & 8000000000000000000000000000000080000000000000000000000000000000 & \\
\hline 36 & & 0000004000002000000008000000000000020008000020008080000000000000 & \\
\hline 50 & & 8000000000000000002200880200200880000000000000000000804000002100 & \\
\hline & 39,30 & $\begin{array}{|llll|}- & 0000080000000000 & - & 8080000000000000 \\
\end{array}$ & \\
\hline 36 & 34,24 & $\begin{array}{llll}- & 0022008802002008 & - & 0000804000002100\end{array}$ & \\
\hline 37 & 13,50 & 808200080000200000000840000420008022008802002008 c000806100002180 & $2^{-18}$ \\
\hline $3 r$ & 10,17 & $8000804000002100882280 a 80288222800000840000020008082000820202000$ & \\
\hline 38 & & 402280e902000188 818a084884040000 082200e802880328 8092480860210104 & $2^{-}$ \\
\hline 30 & & $80820848202000008220 a 0 e 22200 a 1088082084800040000062180$ eb03840188 & \\
\hline
\end{tabular}

